ORIGINAL ARTICLE

\title{
Acute whole body vibration training increases vertical jump and flexibility performance in elite female field hockey players
}

\author{
D J Cochrane, S R Stannard
}

Br J Sports Med 2005;39:860-865. doi: 10.1136/bjsm.2005.019950

See end of article for authors' affiliations

.....................

Correspondence to: Darryl J Cochrane, Massey

University, Sport and Exercise, Palmerston North, New Zealand; d. cochrane@massey.ac.nz

Accepted 11 April 2005
Objective: To quantify the acute effect of whole body vibration (WBV) training on arm countermovement vertical jump (ACMVJ), grip strength, and flexibility performance.

Methods: Eighteen female elite field hockey players each completed three interventions of WBV, control, and cycling in a balanced random manner. WBV was performed on a Galileo machine $(26 \mathrm{~Hz})$ with six different exercises being performed. For the control, the same six exercises were performed at $0 \mathrm{~Hz}$, whilst cycling was performed at $50 \mathrm{~W}$. Each intervention was $5 \mathrm{~min}$ in duration with ACMVJ, grip strength, and flexibility measurements being conducted pre and post intervention.

Results: There was a positive interaction effect (intervention $\times$ pre-post) of enhanced ACMVJ $(p<0.001)$ and flexibility $(p<0.05)$ parameters following WBV; however no changes were observed after the control and cycling interventions. There was no interaction effect for grip strength following the three interventions. Conclusions: Acute WBV causes neural potentiation of the stretch reflex loop as shown by the improved ACMVJ and flexibility performance. Additionally, muscle groups less proportionally exposed to vibration do not exhibit physiological changes that potentiate muscular performance.
W hole body vibration (WBV) is a novel training intervention performed on a commercially manufactured machine known as the Galileo Sport. This instrument was originally developed to increase power and strength in athletes ${ }^{1-3}$ and its vibration production has been described elsewhere. ${ }^{4}$ However, in summary the Galileo Sport machine has a tilting platform that delivers oscillatory movements to the body of varying frequencies $(0-30 \mathrm{~Hz})$ around a horizontal axle. The subject stands on the machine with the feet placed on either side of the axle and maintains a steady position while the oscillations of the platform produce a vertical ground reaction force to each foot alternately. The rapidly repeating eccentric-concentric contractions presumably evoked, result in muscular work evidenced by a significant elevation in metabolic rate. ${ }^{4}$

Proven to be effective in improving strength, ${ }^{56}$ bone density, ${ }^{7}$ and body composition, ${ }^{5}$ this novel training intervention is becoming popular for conditioning, rehabilitation, and general health. However, the acute effects of WBV on power, strength, and flexibility are still largely untested.

Superimposed cable vibration increases maximal muscular force $^{8}$ and an improved residual post-vibration effect has been noted. ${ }^{9-11}$ The latter is suggested to be the joint result of increased sensitivity of muscle stretch receptors to excitation, elevated muscle temperature, and improved blood flow. ${ }^{8}$ The improvement in muscular power following acute exposure results in performance gain that would otherwise require weeks of training. ${ }^{10} 11$

From these observations, it would be expected that a functional measure of muscular power, such as maximal vertical jump height, would be significantly enhanced by acute exposure to WBV. However, the data are equivocal, showing either no change, ${ }^{12}$ a decrease, ${ }^{13}$ or an increase ${ }^{14} 15$ following acute exposure to WBV. Nevertheless, improper control, differences in vibration duration, frequency, and amplitudes, lack of or use of a warm up, and differing exercise positions, may explain the discrepancies in the results. In addition, previous studies have not permitted arm movement in the vertical jump test in an attempt to isolate lower limb muscular performance. In sport, jumping movements generally involve arm drive, so information regarding vertical jump performance with arm countermovement (arm countermovement vertical jump, ACMVJ) after acute WBV is required.

The paucity of well controlled studies investigating the acute effect of WBV on vertical jump height is surprising considering the number of sporting activities which would be enhanced by an improvement in vertical jump height. Furthermore, the lay literature suggests that other measures, such as flexibility, are influenced by acute WBV, but there is little information in the scientific literature in this regard.

Therefore, the primary aim of this study was to quantify the acute effect of WBV on ACMVJ performance in elite female field hockey players. To better understand the mechanism by which WBV may exert an ergogenic effect, a secondary aim of this study was to observe whether muscle groups exposed less proportionally to vibration are capable of enhancing muscular performance. Finally, a third aim was to test the effect of WBV on flexibility using a sit and reach test.

\section{METHODS}

\section{Participants}

Eighteen healthy female elite field hockey players volunteered to participate in the study. They were (mean \pm standard deviation) $21.8 \pm 5.9$ years old and $1.66 \pm 0.06 \mathrm{~m}$ tall, and weighed $63.7 \pm 7.6 \mathrm{~kg}$. The protocol was approved by the Massey University Human Ethics Committee.

\section{Study design}

ACMVJ, isometric grip strength, and sit and reach flexibility were measured before and after the following three interventions, which each lasted 5 min:

Abbreviations: ACMVJ, arm countermovement vertical jump; ANOVA, analysis of variance; GTO, Golgi tendon organ; ICC, intraclass correlation coefficient; WBV, whole body vibration 
- Standing on the Galileo Sport machine with vibration (WBV)

- Standing on the Galileo Sport machine without vibration (control)

- Seated cycling

Within $15 \mathrm{~s}$ of completing the intervention, participants were retested on ACMVJ, grip strength, and flexibility measures in the same order as before the intervention.

The intervention order was allocated in a randomised, balanced design, with $24 \mathrm{~h}$ recovery between each testing session. During the course of the study, participants were not permitted to undertake any power or strength training and timing of the menstrual phase was accounted for. The participants were strictly instructed to refrain from undertaking any vigorous activity $24 \mathrm{~h}$ prior to the interventions, and, to prevent interference from variations in daily biorhythms, participants performed the tests at the same time each day. Each participant was familiarised with the equipment and tests prior to the study and performed pre and post measures of countermovement vertical jump, grip strength, and sit and reach. Every participant wore exactly the same pair of shoes to standardise vibration dampening.

\section{Performance tests}

Warm up was prohibited prior to the performance tests to reduce the possibility of influencing the outcome of the study.

\section{Vertical jump test}

Six countermovement jumps with arm swing (ACMVJ) were performed according to the protocol of Harman et al. ${ }^{16}$ Each jump was recorded to $0.1 \mathrm{~cm}$ and was separated by a rest period of $10 \mathrm{~s}$. The ACMVJ was measured by a system consisting of a portable hand held computer unit connected to a contact timing mat (Swift Performance, NSW, Australia). It has been previously reported that this system has a validity and reliability similar to a force plate. ${ }^{17}$

\section{Hand grip strength test}

Hand grip strength was determined by a hand grip dynamometer (Smedlay, Tokyo). Participants stood upright with arms down the side of the body. The dominant hand performed three repetitions of a $2 \mathrm{~s}$ grip contraction which was recorded to the nearest $0.1 \mathrm{~kg}$.

\section{Sit and reach test}

The sit and reach test was conducted on a sit and reach apparatus (Figure Finder Flex Test, Novel, Rockton, IL) and followed the procedure as outlined by Church et al ${ }^{18}$ with the maximum end position being held for $2 \mathrm{~s}$. Two repetitions were recorded to the nearest centimetre and separated by a rest period of $10 \mathrm{~s}$.

\section{Interventions WBV}

This was performed on a commercial Galileo Sport machine (Novotec, Pforzheim, Germany). The participants stood on the machine and positioned their feet around the centre of the oscillating platform, that equated to a peak to peak amplitude of $6 \mathrm{~mm}$ of vertical vibration. The frequency was set at $26 \mathrm{~Hz}$. The positions taken by the subject were: (1) standing upright with knees semi-locked; (2) isometric squat at a knee angle of approximately $120^{\circ}$; (3) kneeling on the ground with arms straight and hands placed on the platform equating to a peak to peak amplitude of $6 \mathrm{~mm}$ of vertical vibration; (4) squatting at a tempo of $2 \mathrm{~s}$ up and $2 \mathrm{~s}$ down at a knee angle of approximately $120^{\circ}$; (5) lunge position with left leg on platform and right leg on ground; and (6) lunge

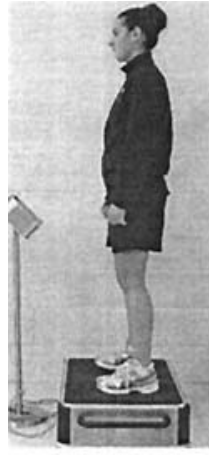

Position 1
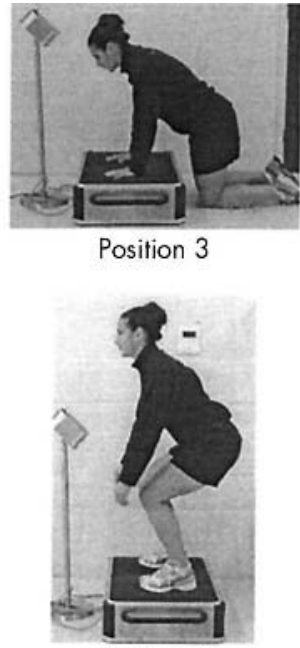

Position 4

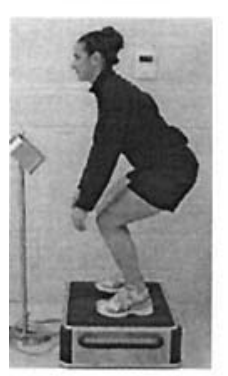

Position 3
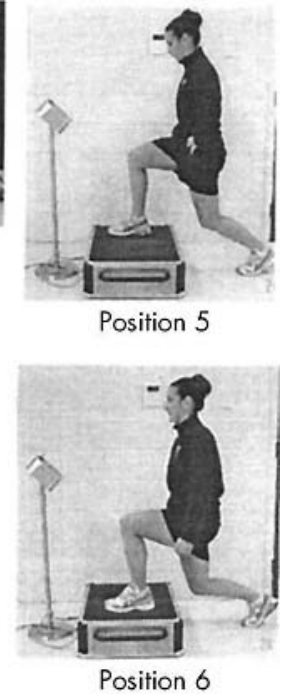

Position 6
Position 2

Figure 1 The six positions performed on the Galileo Sport machine. (The subject depicted in these images agreed to the photographs being published.)

position with right leg on platform and left leg on ground (fig 1). Positions $1-4$ were held for $1 \mathrm{~min}$ and positions 5 and 6 for $30 \mathrm{~s}$.

\section{Control}

The control intervention was performed on the Galileo Sport machine $(0 \mathrm{~Hz}$, amplitude $0 \mathrm{~mm})$ with the exact same six body positions and time constructs as described for the WBV intervention.

\section{Cycling}

In the seated position, the subject pedalled at a cadence of $50 \mathrm{rpm}$ for $5 \mathrm{~min}$ at $50 \mathrm{~W}$ on a friction braked cycle ergometer (Monark 818 E, Varberg, Sweden).

\section{Statistical analysis}

All statistical analyses were performed using a specialised statistical software package (SPSS for Windows version 10; SPSS, Chicago, IL). Dependant variables were compared using a three way, repeated measures analysis of variance (ANOVA). Each test data measure (six for ACMVJ, three for hand grip, and two for sit and reach) was included as part of the ANOVA analysis. Pairwise comparison between means was performed using post hoc contrasts to identify intervention difference. Intraclass correlation coefficients (ICC) assessed the test-retest reliability of comparing the mean of the dependent variables between testing sessions. Significance was considered to be at or greater than the $95 \%$ level of confidence $(p \leqslant 0.05)$.

\section{RESULTS}

\section{ACMV J}

There was a significant $(\mathrm{p}<0.001)$ (intervention $\times$ pre-post) interaction (fig 2) such that WBV produced an ergogenic effect resulting in an $8.1 \pm 5.8 \%$ increase in ACMVJ height (fig 3) compared to both control $(-2.0 \pm 3.7 \%)$ and cycling $(-0.3 \pm 3.7 \%)$. 


\section{Hand grip strength}

There was no significant interaction effect for WBV, control, and cycling interventions (fig 4). There was a significant effect in that each repetition of grip strength decreased $(\mathrm{p}<0.05)$.

\section{Sit and reach}

The interaction between treatment and pre-post sit and reach $(p<0.05)$ was significantly greater after WBV $(8.2 \pm 5.4 \%)$ compared to the control and cycling interventions $(5.3 \pm 5.1 \%$ and $5.3 \pm 4.9 \%$, respectively; fig 5 ). Post hoc analysis revealed that for all interaction interventions (WBV, control, and cycling) sit and reach increased for each repetition $(\mathrm{p}<0.001)$. The ICC test-retest reliability analyses revealed that jump height $(0.916)$, grip strength $(0.804)$, and sit and reach $(0.934)$ were consistent between sessions.

\section{DISCUSSION}

\section{ACMVJ}

The primary purpose of this study was to examine the acute effects of WBV (eccentric-concentric loading), control, and cycling (concentric only loading) interventions on ACMVJ performance. Our results show that ACMVJ performance is enhanced by $8.1 \%$ immediately following $5 \mathrm{~min}$ of WBV exposure when compared to control (no vibration) conditions. These observations are in accordance with the findings of Bosco et al ${ }^{14}$ and Torvinen et $a l^{15}$ who reported increases of $2.5 \%$ and $3.8 \%$ in vertical jump height, respectively, but without arm action.

The ACMVJ is a common test used by conditioners and coaches in a variety of game sports to monitor the effects of training and/or rehabilitation. Additionally, it is an established measure of lower body explosive power and, as such, it should be used as part of a battery of tests for assessing the field hockey player. ${ }^{19}$ Although the $\sim 8 \%$ improvement in ACMVJ height seen in this study cannot be directly extrapolated to predict field hockey performance, it would have relevance in game situations such as changing direction, lunging, and acceleration, where maximal explosive power is important. In this context, only small enhancements in muscular performance are needed to provide the necessary edge in elite competition.

The fact that the protocol of the present study enhanced jump performance to a greater degree than in previous studies using an acute WBV intervention is difficult to explain. However, the pre-intervention measures of ACMVJ in the present study were performed without a warm up, whereas the Bosco et $a l^{14}$ and Torvinen et al ${ }^{15}$ studies which

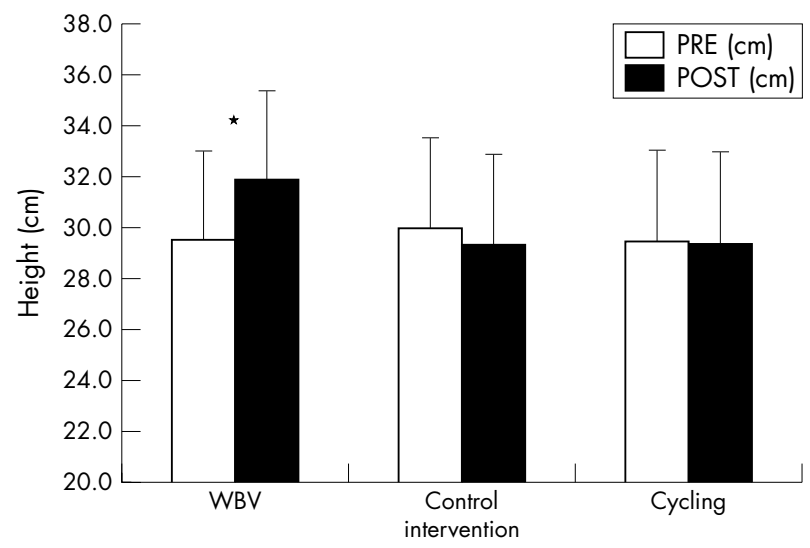

Figure 2 Mean and standard deviation pre and post ACMVJ for WBV, control, and cycling interventions. *Statistically significant $(p<0.001)$ interaction effect (pre-post) for WBV intervention. revealed a smaller ergogenic effect of WBV, employed a cycling warm up prior to the first measure. Although in the present study the effect of seated cycling alone on ACMVJ did not reach significance ( $p=0.07$ ), it is likely that, with greater participant numbers, we would have observed improved jump performance compared to control conditions. Thus, it is possible that cycling alone may provide sufficient warm up to enhance vertical jump performance to a small degree, and this may explain the smaller ergogenic effects of the aforementioned studies in which cycling exercise was used as a warm up. It is clear, however, from the results of the present study and those of Bosco et al ${ }^{14}$ and Torvinen et al, ${ }^{15}$ that the effects of vibration are additive to any cycling based warm up.

In the present study different muscle contractions were utilised in the different interventions: WBV exposure elicits both concentric and eccentric contractions, whilst seated cycling requires only concentric muscle action. ACMVJ involves activation of the stretch-shortening cycle, where the stretch receptors are activated under the eccentric loading phase. Given the significant enhancement of ACMVJ by WBV compared to cycling, it could be speculated that the additional effect of WBV over cycling was due to the eccentric stimuli it provides.

The enhanced muscle power observed following acute vibration is suggested to occur via potentiation of the neuromuscular system whereby stimulation of muscles spindles (Ia afferents) results in reflex activation of motor neurones with increased spatial recruitment. ${ }^{20} 21$ The continued enhancement of the stretch-reflex pathway can also be attributed to the $\gamma$ motor neurone input causing an increase in sensitivity of the primary endings. Furthermore, the tonic vibration reflex can recruit additional motor units via muscle spindle and polysynaptic activation. ${ }^{15}$

This current study did not include EMG recordings and therefore it is not possible to directly assess any neurogenic enhancement. However, it has been reported that the stretchshortening cycle of the ACMVJ activates the spinal reflexes to enhance jump height compared to that of a concentric squat jump. ${ }^{22}$ Therefore, it is reasonable to propose that any neurogenic changes are ably detected by ACMVJ. The accentuated jump height following acute WBV suggests that neural enhancement occurred through an increased sensitivity of the stretch reflex mechanism and is in agreement with other investigators. ${ }^{310142324}$ However, in the absence of measures of neural function, such an explanation remains speculative and a better understanding awaits further investigation.

The use of arm countermovement in the vertical jump measure in the present study is unlikely to have contributed to the greater enhancement of jump performance, unless WBV somehow improves the coordination of arm and leg movements. Additionally, arm swing in the countermovement jump has been proven to require less practice than a countermovement jump with no arm swing and has been described as a natural practiced movement. ${ }^{19}$

\section{Hand grip strength}

Maximal grip strength was not improved with either intervention compared to control conditions. The level of vibration exposure sustained by the forearm muscles during the protocol in the present study is difficult to quantify, but would have been small. The results confirm the findings of Torvinen et al, ${ }^{15}$ illustrating the fact that muscles not directly exposed to vibration do not show a concomitant performance enhancement as does the vibrated muscle. Therefore, the improved ACMVJ performance seen in the present study is not related to a vibration induced centrally 


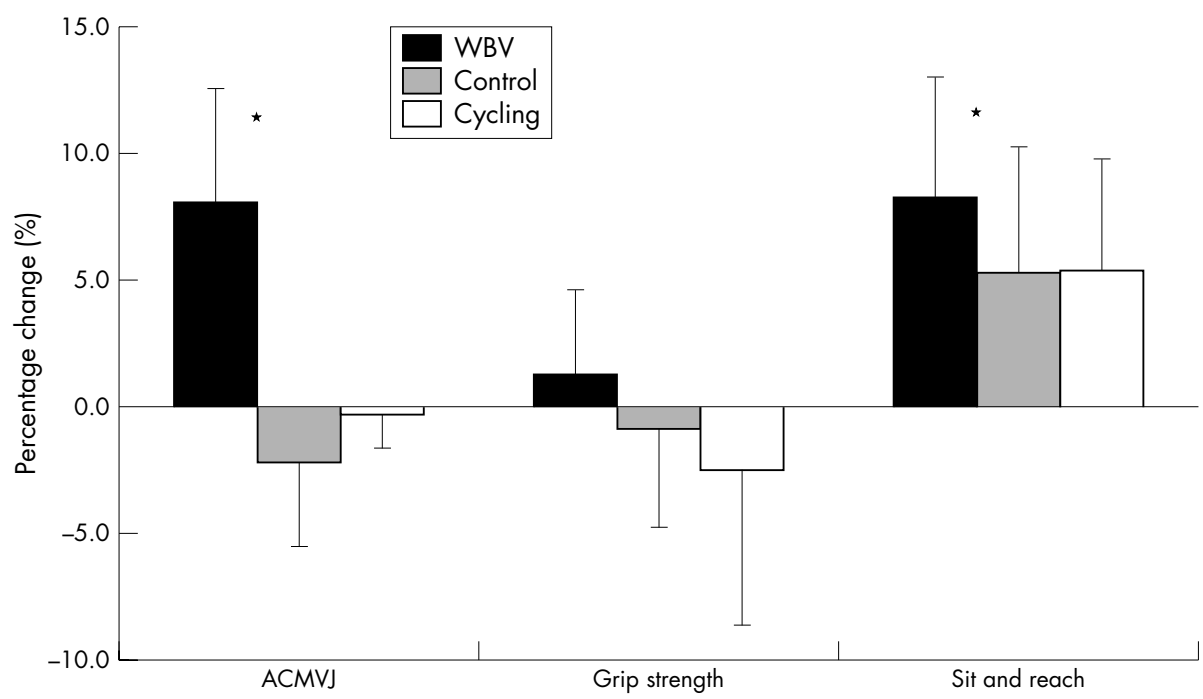

Figure 3 Percentage change in ACMVJ, grip strength, and sit and reach for WBV, control, and cycling interventions. *Statistically significant $(p<0.001)$ percentage change pre-post interaction.

mediated phenomenon but confirms that the effect is localised to the spinal level and/or the muscle itself.

\section{Flexibility}

The significant improvement of $8.2 \%$ in the sit and reach flexibility following WBV is comparable to the $8 \%$ increase in leg split flexibility reported by Issurin et al. ${ }^{2}$ However, their protocol of flexibility training was performed with a vibrating cable simultaneously attached to the lower limb of the participant. ${ }^{2}$ In the present study, no flexibility exercises were conducted concurrently with the three interventions.

As stated previously, vibration enhances the stretch reflex loop through the activation of the primary endings of the muscle spindle, which influences agonist muscle contraction while antagonists are simultaneously inhibited. ${ }^{25}$ The enhanced flexibility measure following WBV was greater than that after the control and cycling interventions, which suggests that the vibration exposure may have activated the Ia inhibitory interneurones of the antagonist muscle. This in turn may have caused changes to intramuscular coordination to decrease the braking force around the hip and lower back joints and potentiate the sit and reach score. ${ }^{3}$

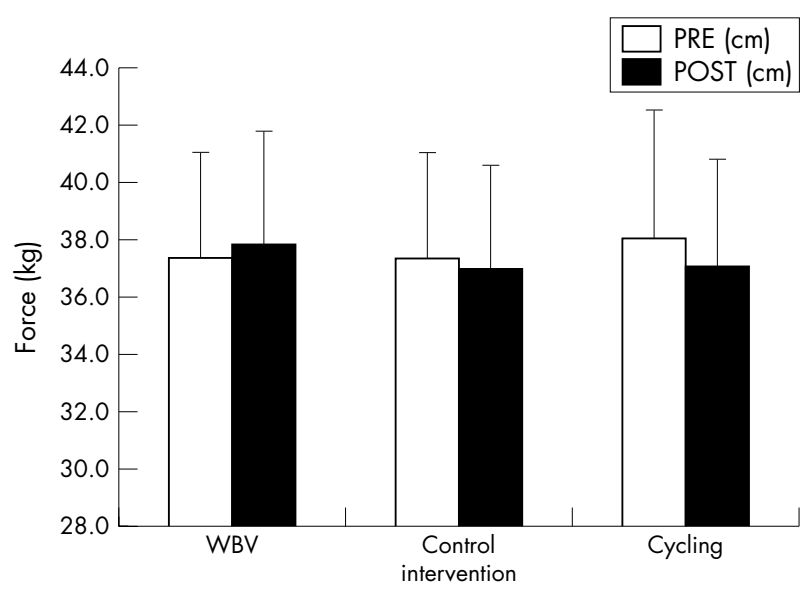

Figure 4 Mean and standard deviation pre and post grip strength for WBV, control, and cycling interventions.
Increases in static and dynamic muscular contractions have been attributed to muscle stiffness, which is a function of muscle and tendon components. ${ }^{26}$ The magnitude of the stretch load and the condition of the musculotendinous complex ultimately determine which reflexes dominate. ${ }^{27}$ For pre-stretching to enhance concentric muscular contraction, excitatory responses of the muscle spindle must exceed the inhibitory effects of the Golgi tendon organ (GTO). This is normally achieved through potentiated neural input of muscle spindle sensitivity or suppression of GTO neural activity.

In strength and power training, performing heavy sets of squats has been shown to augment jump squat height. ${ }^{28}$ Equally in WBV, fast joint rotation and muscle stretching occur, which is likely to increase muscle stiffness following the purported neural potentiation of the stretch reflex pathway and motor neurone input. ${ }^{29}$ Moreover, vibration causes more excitatory responses to the primary endings of muscle spindles compared to secondary endings and GTOs. The joint, skin, and secondary endings also detect the vibratory stimulus whereby the neural activity of the primary endings is potentiated through the activation of the $\gamma$ motor

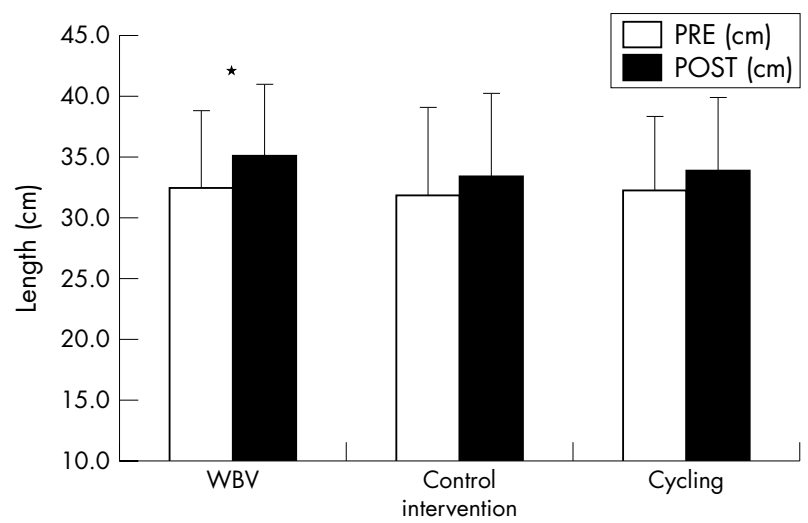

Figure 5 Mean and standard deviation pre and post sit and reach for WBV, control, and cycling interventions. *Statistically significant $(p<0.001)$ interaction effect (pre-post) for WBV, control, and cycling interventions. 


\section{What is already known on this topic}

Whole body vibration (WBV) has proven to be effective in improving strength, power, and bone density. This novel training intervention is becoming popular for conditioning, rehabilitation, and general health. However, the acute effects of WBV on power, strength, and flexibility are still largely untested.

neurone. ${ }^{3}$ This post activation potentiation, known in the strength conditioning field as "tuning up", may explain the concomitant enhancement of ACMVJ and flexibility performance.

The vibratory stimulus of the Ia neural drive and proprioceptive loop may also replicate a warm up effect by increasing pain threshold, blood flow, and muscle elasticity. ${ }^{2}$ Kerschan-Schindl et $a l^{30}$ have reported an increase in mean blood flow of the popliteal artery after acute WBV. They cite a combination effect of possible vasodilation and thixotropism for reducing the viscosity of the blood and improving the mean speed of blood flow. Acute vibration exposure has also been shown to reduce pain affected areas of muscle or tendon, ${ }^{31}$ which may allow a greater tolerance of the stretch threshold.

As yet, there are no set guidelines for WBV exercises, hence different investigators have used very different protocols $^{141532}$ with few comparisons with other controls. In this study, WBV elicited a greater increase in ACMVJ and flexibility compared to cycling, which suggests that WBV may be an effective intervention for warming up. Numerous warm up mechanisms have been described to increase performance, ${ }^{33}$ therefore it is difficult to explain how WBV may accelerate the warm up effect. However, given that WBV results in concentric-eccentric contraction but cycling in solely concentric, WBV may provide an additional eccentric stimulus that is currently overlooked in conventional warm up procedures. Incorporating a greater eccentric component in warm ups may be beneficial for enhancing performance in sporting activities that rely on the eccentric-concentric interaction, which with WBV requires little effort and time. Further investigation is required before any conclusion can be drawn that acute WBV may be used as a potential warm up intervention.

\section{Conclusions}

In conclusion, this study further substantiates the claims of other investigators ${ }^{14} 1^{34}$ that acute WBV causes neural potentiation of the stretch reflex loop as observed by the improved ACMVJ and flexibility performance. Additionally, muscle groups less proportionally exposed to vibration do not exhibit physiological changes that potentiate muscular performance.

\section{Authors' affiliations \\ D J Cochrane, S R Stannard, Massey University, Palmerston North, New Zealand}

Competing interests: none declared

The subject shown in figure 1 agreed to these photographs being published

\section{REFERENCES}

1 Nazarov V, Spivak G. Development of athlete's strength abilities by means of biomechanical stimulation method. Theory Prac Phys Culture 1985; 12:445-50
What this study adds

This study confirms that power and flexibility increase after acute WBV exposure and that acute WBV may accelerate the warm up effect. Additionally, muscle groups less proportionally exposed to vibration do not exhibit physiological changes that potentiate muscular performance.

2 Issurin VB, Liebermann DG, Tenenbaum G. Effect of vibratory stimulation training on maximal force and flexibility. J Sports Sci 1994;12:561-6.

3 Cardinale M, Bosco C. The use of vibration as an exercise intervention. Exerc Sport Sci Rev 2003;31:3-7.

4 Rittweger J, Schiesel H, Felsenberg D. Oxygen uptake during whole-body vibration exercise: comparison with squatting as a slow voluntary movement. Eur J Appl Physiol 2001;86:169-73.

5 Roelants M, Delecluse C, Goris M, et al. Effects of 24 weeks of whole body vibration training on body composition and muscle strength in untrained females. Int J Sports Med 2004;25:1-5.

6 Roelants M, Delecluse C, Verschueren S. Whole body vibration increases knee extension strength and speed of movement in older women. J Am Geriatr Soc 2004;52:901-8.

7 Verschueren SMP, Roelants M, Delecluse $C$, et al. Effect of 6-month whole body vibration training on hip density, muscle strength, and postural control in postmenopausal women: a randomized controlled pilot study. J Bone Miner Res 2004; 19:352-9.

8 Issurin VB, Tenenbaum G. Acute and residual effects of vibratory stimulation on explosive strength in elite and amateur athletes. J Sports Sci 1999;17:177-82.

9 Cafarelli E, Laytonwood J. Effect of vibration on force sensation in fatigued muscle. Med Sci Sport Exerc 1986;18:516-21.

10 Bosco C, Cardinale M, Tsarpela O, et al. New trends in training science: the use of vibrations for enhancing performance. New Stud Athletics 1999; 14:55-62.

11 Bosco C, Cardinale M, Tsarpela O. Influence of vibration on mechanical power and electromyogram activity in human arm flexor muscles. Eur J Appl Physiol 1999;79:306-11.

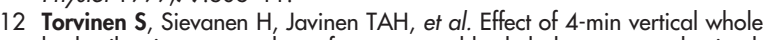
body vibration on muscle performance and body balance: a randomized cross-over study. Int J Sports Med 2002;23:374-9.

13 Bosco C, Colli O, Cardinale M, et al. The effects of whole body vibration on mechanical behaviour of skeletal muscle and hormonal profile. In: GP Lyritis, ed. Musculoskeletal interactions. Basic and clinical aspects. Vol 2. Athens: Hylonome, 1999:67-76.

14 Bosco C, lacovelli M, Tsarpela O, et al. Hormonal responses to whole-body vibration in men. Eur J Appl Physiol 2000;81:449-54.

15 Torvinen S, Kannus P, Sievanen H, et al. Effect of a vibration exposure on muscular performance and body balance. Randomized cross-over study. Clin Physiol Funct Imaging 2002;22:145-52.

16 Harman EA, Rosenstein MT, Frykman PN, et al. The effects of arms and countermovement on vertical jumping. Med Sci Sports Exerc 1990;22:825-33.

17 Cronin JB, McNair PJ, Marshall RJ. Relationship between strength qualities and motor skills associated with court performance. J Hum Mov Stud 2001;40:207-24.

18 Church JB, Wiggins MS, Moode FM, et al. Effect of warm-up and flexibility treatments on vertical jump performance. J Strength Cond Res 2001;15:332-6.

19 Keogh JWL, Weber CL, Dalton CT. Evaluation of anthropometric, physiological, and skill-related tests for talent identification in female field hockey. Can J Appl Phys 2003;28(3):397-409.

20 Romaiguere $\mathbf{P}$, Vedel JP, Pagni S. Effects of tonic vibration reflex on motor unit recruitment in human wrist extensor muscles. Brain Res 1993;602:32-40

21 Komi PV. Stretch-shortening cycle: a powerful model to study normal and fatigued muscle. J Biomech 2000;33:1197-1206.

22 Bobbert MF, Gerritsen KGM, Litjens MCA, et al. Why is countermovement jump height greater than squat jump height? Med Sci Sports Exerc 1996;28:1402-12.

23 de Ruiter CJ, van der Linden RM, van der Zijden MJA, et al. Short-term effects of whole-body vibration on maximal voluntary isometric knee extensor force and rate of force rise. Eur J Appl Physiol 2003;88:472-5.

24 Delecluse C, Roelants $M$, Verschueren S. Strength increase after whole-body vibration compared with resistance training. Med Sci Sports Exerc 2003;35: 1033-41.

25 Rothmuller C, Cafarelli E. Effect of vibration on antagonist muscle coactivation during progressive fatigue in humans. J Physiol 1995;485:857-64

26 Ettema GJC, Huijing PA. Skeletal muscle stiffness in static and dynamic contractions. J Biomech 1994;27:1361-8.

27 Lambertz D, Goubel F, Kaspranski R, et al. Influence of long-term spaceflight on neuromechanical properties of muscles in humans. J App Physiol 2003;94:490-8.

28 Barker D. Selecting the appropriate exercises and loads for speed-strength development. Strength Cond Coach 1995;3:8-14. 
29 Cardinale $M$, Lim J. The acute effects of two different whole body vibration frequencies on vertical jump performance. Med Del Sport 2003;56:287-92.

30 Kerschan-Schindl K, Grampp S, Henk C, et al. Whole-body vibration exercise leads to alterations in muscle blood volume. Clin Physiol 2001;21:377-82.

31 Lundeberg T, Nordemar R, Ottoson D. Pain alleviation by vibratory stimulation. Pain 1984;20:25-44.
32 Bosco C, Cardinale M, Colli O, et al. The influence of whole body vibration on jumping performance. Biol Sport 1998;15:157-64.

33 Bishop D. Warm up II: performance changes following active warm up and how to structure the warm up. Sports Med 2003;33:483-98.

34 Rittweger J, Beller G, Felsenberg D. Acute physiological effects of exhaustive whole-body vibration exercise in man. Clin Physiol 2000;20:134-42.

\section{Clinical Evidence-Call for contributors}

Clinical Evidence is a regularly updated evidence-based journal available worldwide both as a paper version and on the internet. Clinical Evidence needs to recruit a number of new contributors. Contributors are healthcare professionals or epidemiologists with experience in evidence-based medicine and the ability to write in a concise and structured way.

Areas for which we are currently seeking authors:

- Child health: nocturnal enuresis

- Eye disorders: bacterial conjunctivitis

- Male health: prostate cancer (metastatic)

- Women's health: pre-menstrual syndrome; pyelonephritis in non-pregnant women However, we are always looking for others, so do not let this list discourage you.

Being a contributor involves:

- Selecting from a validated, screened search (performed by in-house Information Specialists) epidemiologically sound studies for inclusion.

- Documenting your decisions about which studies to include on an inclusion and exclusion form, which we keep on file.

- Writing the text to a highly structured template (about 1500-3000 words), using evidence from the final studies chosen, within 8-10 weeks of receiving the literature search.

- Working with Clinical Evidence editors to ensure that the final text meets epidemiological and style standards.

- Updating the text every six months using any new, sound evidence that becomes available. The Clinical Evidence in-house team will conduct the searches for contributors; your task is simply to filter out high quality studies and incorporate them in the existing text.

- To expand the topic to include a new question about once every 12-18 months.

If you would like to become a contributor for Clinical Evidence or require more information about what this involves please send your contact details and a copy of your CV, clearly stating the clinical area you are interested in, to Klara Brunnhuber (kbrunnhuber@ bmigroup.com).

\section{Call for peer reviewers}

Clinical Evidence also needs to recruit a number of new peer reviewers specifically with an interest in the clinical areas stated above, and also others related to general practice. Peer reviewers are healthcare professionals or epidemiologists with experience in evidence-based medicine. As a peer reviewer you would be asked for your views on the clinical relevance, validity, and accessibility of specific topics within the journal, and their usefulness to the intended audience (international generalists and healthcare professionals, possibly with limited statistical knowledge). Topics are usually 1500-3000 words in length and we would ask you to review between 2-5 topics per year. The peer review process takes place throughout the year, and our turnaround time for each review is ideally 10-14 days.

If you are interested in becoming a peer reviewer for Clinical Evidence, please complete the peer review questionnaire at www.clinicalevidence.com or contact Klara Brunnhuber (kbrunnhuber@bmigroup.com). 